\title{
Existence and multiplicity of difference $\phi$-Laplacian boundary value problems
}

\section{Dingyong Bai ${ }^{*}$ and Xianliang Xu}

\section{"Correspondence:}

baidy@gzhu.edu.cn

School of Mathematics and

Information Science, and Key

Laboratory of Mathematics and

Interdisciplinary Sciences of

Guangdong Higher Education

Institutes, Guangzhou University,

Guangzhou, 510006, P.R. China

\begin{abstract}
Concerned are the difference $\phi$-Laplacian boundary value problems. The multiplicity result based on the lower and upper solutions method associated with Brouwer degree is applied to a difference $\phi$-Laplacian eigenvalue problem. An existence result of at least three positive solutions is established for the eigenvalue problem with the parameter belonging to an explicit open interval. In addition, an example is given to illustrate the three solutions result.
\end{abstract}

Keywords: difference $\boldsymbol{\phi}$-Laplacian boundary value problem; the lower and upper solution; positive solution; multiplicity; Brouwer degree

\section{Introduction}

Recently, Kim [1] studied a one-dimensional differential $p$-Laplacian boundary value problem with a positive parameter and established an existence result of three positive solutions by the lower and upper solutions method associated with Leray-Schauder degree theory. Kim and Shi [2] studied the global continuum and multiple positive solutions of a $p$-Laplacian boundary value problem. Motivated by the methods in $[1,2]$, we consider difference $\phi$-Laplacian boundary value problems.

For $a, b \in \mathbf{Z}$ with $a<b$, let $[a, b]_{\mathbf{Z}}=\{a, a+1, a+2, \ldots, b-1, b\}$. First, by the upper and lower solutions method associated with Brouwer degree theory, we establish the existence and multiplicity results for the following discrete $\phi$-Laplacian boundary value problem:

$$
\left\{\begin{array}{l}
\Delta(\phi(\Delta u(k-1)))+f(k, u(k))=0, \quad k \in[1, T]_{\mathrm{Z}}, \\
u(0)=u(T+1)=0,
\end{array}\right.
$$

where $T>1$ is a given positive integer, $\Delta u(k)=u(k+1)-u(k)$, and

(A1) $\phi: \mathbf{R} \rightarrow \mathbf{R}$ is an odd and strictly increasing function;

(A2) $f:[1, T]_{\mathbf{Z}} \times \mathbf{R} \rightarrow \mathbf{R}$ is continuous.

Then, we apply the multiplicity result of (1) to the following eigenvalue problem:

$$
\left\{\begin{array}{l}
\Delta(\phi(\Delta u(k-1)))+\lambda p(k) g(u(k))=0, \quad k \in[1, T]_{\mathrm{Z}}, \\
u(0)=u(T+1)=0
\end{array}\right.
$$

where $\lambda$ is a positive parameter. Under some suitable assumptions imposed on $g$, we establish the existence of three positive solutions of (2) with $\lambda$ belonging to an explicit open interval.

(c) 2013 Bai and Xu; licensee Springer. This is an Open Access article distributed under the terms of the Creative Commons Attribution License (http://creativecommons.org/licenses/by/2.0), which permits unrestricted use, distribution, and reproduction in any medium, provided the original work is properly cited. 
The function $\phi(u)$ covers two important cases: $\phi(u)=u$ and $\phi(u)=|u|^{p-2} u(p>1)$. If $\phi(u)=u$, then problem (1) is the classical second order difference Dirichlet boundary value problem. For the case that $\phi(u)=|u|^{p-2} u$, problem (1) is the well-known discrete $p$-Laplacian problem. The two cases have been widely studied. To name a few, see [3-10] and the references therein.

Problem (1) can be viewed as the discrete analogue of the following differential $\phi$ Laplacian problem:

$$
\left\{\begin{array}{l}
\left(\phi\left(u^{\prime}\right)\right)^{\prime}+f(t, u)=0, \quad 0<t<1 \\
u(0)=u(1)=0
\end{array}\right.
$$

which rises from the study of radial solutions for $p$-Laplacian equations $\left(\phi(u)=|u|^{p-2} u\right)$ on an annular domain (see [11], and references therein). Recently, the differential $\phi$-Laplacian problems have been widely studied in many different papers. We refer the readers to [12$19]$ and the references therein.

For discrete $\phi$-Laplacian problems, there are fewer study results than for differential $\phi$ Laplacian problems. See Cabda [20], Cabada and Espinar [21] and Bondar [22]. To the best of our knowledge, there are no results on the existence and multiplicity of positive solutions for difference $\phi$-Laplacian problems.

The remaining part of this paper is organized as follows. In Section 2, we show the lower and upper solutions method and establish the existence and multiplicity of solutions of (1). In Section 3, we establish the existence of three positive solutions of (2). Finally, we give an example to illustrate our main results.

\section{The upper and lower solutions method}

In this section, we establish the existence and multiplicity results of solutions for problem (1) by lower and upper solutions method associated with Brouwer degree.

Let $E=\left\{u:[0, T+1]_{\mathbf{Z}} \rightarrow \mathbf{R}^{T+2}\right\}$ with the norm $\|u\|=\max _{t \in[0, T+1]_{\mathbf{Z}}}|u(t)|$.

Definition 2.1 Given $u, v, w \in E$, we say that

(1) $u \leq v$ if for all $k \in[0, T+1]_{\mathbf{Z}}, u(k) \leq v(k)$.

(2) $u \in[v, w]$ if $v \leq u \leq w$.

(3) $u \prec v$ if for all $k \in[1, T]_{\mathrm{Z}}, u(k)<v(k)$ and $u(0) \leq v(0), u(T+1) \leq v(T+1)$.

Definition 2.2 $\alpha \in E$ is called a lower solution of problem (1) if

$$
\left\{\begin{array}{l}
\Delta(\phi(\Delta \alpha(k-1)))+f(k, \alpha(k)) \geq 0, \quad k \in[1, T]_{\mathrm{Z}} \\
\alpha(0) \leq 0, \quad \alpha(T+1) \leq 0
\end{array}\right.
$$

If the first inequality above is strict, then $\alpha$ is called a strict lower solution of (1).

In the same way, we define the upper solution and the strict upper solution of (1) by reversing the inequalities above.

Lemma 2.1 Let (A1) hold. The problem

$$
\left\{\begin{array}{l}
-\Delta(\phi(\Delta u(k-1)))+u(k)=0, \quad k \in[1, T]_{\mathrm{Z}} \\
u(0)=u(T+1)=0
\end{array}\right.
$$

has the unique solution $u \equiv 0$. 
Proof It is clear that 0 is a trivial solution of (4). Suppose that (4) has a nontrivial solution $u$. Let $|u(m)|=\max _{k \in[1, T]_{\mathbf{Z}}}|u(k)|=\|u\|$. If $u(m)=\max _{k \in[1, T]_{\mathbf{Z}}} u(k)$, then $u(m)>0$ and $\Delta u(m) \leq$ $0, \Delta u(m-1) \geq 0$, which yields a contradiction:

$$
\begin{aligned}
u(m) & =\Delta(\phi(\Delta u(m-1))) \\
& =\phi(\Delta u(m))-\phi(\Delta u(m-1)) \leq 0<u(m) .
\end{aligned}
$$

Similarly, if $u(m)=\min _{k \in[1, T] z} u(k)$, then $u(m)<0$ and $\Delta u(m) \geq 0, \Delta u(m-1) \leq 0$, which implies that $u(m)=\Delta(\phi(\Delta u(m-1))) \geq 0>u(m)$, which is a contradiction. The proof is complete.

Theorem 2.1 Let (A1) and (A2) hold.

(i) Assume that there exist $\alpha$ and $\beta$, respectively lower and upper solutions of (1) such that $\alpha \leq \beta$. Then problem (1) has at least one solution $u$ with $\alpha \leq u \leq \beta$.

(ii) Assume that problem (1) has two pairs of lower and upper solutions $\left(\alpha_{1}, \beta_{1}\right)$ and $\left(\alpha_{2}, \beta_{2}\right)$ with $\alpha_{2}$ and $\beta_{1}$ being strict, satisfying that

$$
\alpha_{1} \leq \alpha_{2} \leq \beta_{2}, \quad \alpha_{1} \leq \beta_{1} \leq \beta_{2}
$$

and that there exists $k_{0} \in[0, T+1]_{\mathrm{Z}}$ such that $\beta_{1}\left(k_{0}\right)<\alpha_{2}\left(k_{0}\right)$. Then problem (1) has at least three solutions $u_{1}, u_{2}, u_{3}$ with

$$
\alpha_{1} \leq u_{1} \prec \beta_{1}, \quad \alpha_{2} \prec u_{2} \leq \beta_{2}, \quad u_{3} \in\left[\alpha_{1}, \beta_{2}\right] \backslash\left(\left[\alpha_{1}, \beta_{1}\right] \cup\left[\alpha_{2}, \beta_{2}\right]\right) .
$$

Remark We denote that the result (i) has been proved in [20] by Brouwer fixed point theorem. Here, for the convenience of the proof of (ii), it is proven by Brouwer degree theory. The proof of (ii) is motivated by the idea in [1].

Proof of Theorem 2.1. (i) Define $\gamma:[1, T]_{\mathbf{Z}} \times \mathbf{R} \rightarrow \mathbf{R}$ by

$$
\gamma(k, u)= \begin{cases}\beta(k), & u>\beta(k) \\ u, & \alpha(k) \leq u \leq \beta(k) \\ \alpha(k), & u<\alpha(k)\end{cases}
$$

Consider the modified problem

$$
\left\{\begin{array}{l}
\Delta(\phi(\Delta u(k-1)))+f(k, \gamma(k, u(k)))-[u(k)-\gamma(k, u(k))]=0, \quad k \in[1, T], \\
u(0)=u(T+1)=0 .
\end{array}\right.
$$

Clearly, all solutions $u$ of (5) satisfying $\alpha \leq u \leq \beta$ are solutions of (1). Let $u$ be a solution of (5). By the arguments in [20], we know that $\alpha \leq u \leq \beta$. Now, we prove that problem (5) has at least one solution. Let $E_{0}=\{u \in E: u(0)=u(T+1)=0\}$ and define operator $\widetilde{T}: E_{0} \rightarrow \mathbf{R}^{T}$ by

$$
(\widetilde{T} u)(k)=\Delta(\phi(\Delta u(k-1)))+f(k, \gamma(k, u(k)))-[u(k)-\gamma(k, u(k))], \quad k \in[1, T]_{\mathbf{Z}}
$$


Obviously, each solution $u$ of $\widetilde{T} u=0$ solves (5). Define homotopic mapping $\Gamma:[0,1] \times$ $E_{0} \rightarrow \mathbf{R}^{T}$ by

$$
\begin{aligned}
\Gamma(\lambda, u)(k) & =(1-\lambda)[\Delta(\phi(\Delta u(k-1)))-u(k)]+\lambda \widetilde{T} u(k) \\
& =\Delta(\phi(\Delta u(k-1)))-u(k)+\lambda[f(k, \gamma(k, u(k)))+\gamma(k, u(k))], \quad k \in[1, T]_{\mathbf{Z}}
\end{aligned}
$$

By the definition of $\gamma$ and the continuity of $f$, there exists an $R>0$, such that

$$
\max _{k \in[1, T]_{\mathbf{Z}}} \max _{u \in \mathbf{R}}|f(k, \gamma(k, u))+\gamma(k, u)|<R .
$$

Let $B_{R}(0)=\left\{u \in E_{0}:\|u\|<R\right\}$. We prove that if $(\lambda, u) \in[0,1] \times E_{0}$ is a solution of $\Gamma(\lambda, u)=0$, then $u \in B_{R}(0)$. Let $|u(m)|=\max _{k \in[1, T]_{\mathbf{Z}}}|u(k)|=\|u\|$. Then there are two cases that $u(m)=\max _{k \in[1, T]_{Z}} u(k)$ and $u(m)=\min _{k \in[1, T]_{\mathbf{Z}}} u(k)$. For the first case, since $u(m+1)-u(m) \leq 0, u(m)-u(m-1) \geq 0$ and $\phi$ is odd, we have that

$$
\begin{aligned}
u(m) & -\lambda[f(m, \gamma(m, u(m)))+\gamma(m, u(m))] \\
= & \Delta(\phi(\Delta u(m-1))) \\
= & \phi(u(m+1)-u(m))-\phi(u(m)-u(m-1)) \\
& \leq 0,
\end{aligned}
$$

which implies that

$$
u(m) \leq \lambda[f(m, \gamma(m, u(m)))+\gamma(m, u(m))]<R .
$$

Similarly, for the second case, we have that

$$
u(m) \geq \lambda[f(m, \gamma(m, u(m)))+\gamma(m, u(m))]>-R .
$$

Therefore, $\|u\|<R$, and $\operatorname{deg}\left(\Gamma(\lambda, \cdot), B_{R}(0), 0\right)$ is well defined. By the homotopy invariance of Brouwer degree, we get that

$$
\operatorname{deg}\left(\widetilde{T}, B_{R}(0), 0\right)=\operatorname{deg}\left(\Gamma(1, \cdot), B_{R}(0), 0\right)=\operatorname{deg}\left(\Gamma(0, \cdot), B_{R}(0), 0\right) .
$$

By Lemma 2.1, the equation $-\Delta(\phi(\Delta u(k-1)))+u(k)=0$ has the unique solution $u=0$ in $E_{0}$, thus we have

$$
\operatorname{deg}\left(\Gamma(0, \cdot), B_{R}(0), 0\right)=1
$$

Therefore, $\operatorname{deg}\left(\widetilde{T}, B_{R}(0), 0\right)=1$, which implies that problem (5) has at least one solution $u \in E_{0}$.

(ii) First, we show that if $\alpha$ and $\beta$ are strict lower and upper solutions, respectively, such that $\alpha \leq \beta$, then $\operatorname{deg}\left(\widetilde{T}, \Omega_{\alpha \beta}, 0\right)=1$, where $\Omega_{\alpha \beta}=\left\{u \in E_{0}, \alpha \prec u \prec \beta,\|u\|<R\right\}$. By the arguments above, each solution $u$ of (5) satisfies that $\alpha \leq u \leq \beta$. We claim that $\alpha \prec u \prec \beta$. 
In fact, if it is not true, then there exists an $m \in[1, T]_{\mathrm{Z}}$ such that $\alpha(m)=u(m)$. Since $\Delta u(m-1) \leq \Delta \alpha(m-1), \Delta u(m) \geq \Delta \alpha(m)$, we have by the monotonicity of $\phi$ that

$$
\begin{aligned}
& \Delta(\phi(\Delta u(m-1)))-\Delta(\phi(\Delta \alpha(m-1))) \\
& \quad=[\phi(\Delta u(m))-\phi(\Delta \alpha(m))]+[\phi(\Delta \alpha(m-1))-\phi(\Delta u(m-1))] \\
& \quad \geq 0 .
\end{aligned}
$$

It yields a contradiction:

$$
\begin{aligned}
\Delta(\phi(\Delta u(m-1))) & =-f(m, \gamma(m, u(m)))+[u(m)-\gamma(m, u(m))] \\
& =-f(m, \alpha(m))<\Delta(\phi(\Delta \alpha(m-1))) .
\end{aligned}
$$

Thus $\alpha \prec u$. Similarly, one can check that $u \prec \beta$. By the excision property of Brouwer degree,

$$
\operatorname{deg}\left(\widetilde{T}, \Omega_{\alpha \beta}, 0\right)=\operatorname{deg}\left(\widetilde{T}, B_{R}(0), 0\right)=1
$$

Now, consider the following modified problem:

$$
\left\{\begin{array}{l}
\Delta(\phi(\Delta u(k-1)))+f\left(k, \gamma^{*}(k, u(k))\right)-\left[u(k)-\gamma^{*}(k, u(k))\right]=0, \quad k \in[1, T], \\
u(0)=u(T+1)=0
\end{array}\right.
$$

where $\gamma^{*}:[1, T]_{\mathbf{Z}} \times \mathbf{R} \rightarrow \mathbf{R}$ is defined by

$$
\gamma^{*}(k, u)= \begin{cases}\beta_{2}(k), & u>\beta_{2}(k) \\ u, & \alpha_{1}(k) \leq u \leq \beta_{2}(k) \\ \alpha_{1}(k), & u<\alpha_{1}(k)\end{cases}
$$

It is easy to see that for sufficiently small $\epsilon>0,\left(\alpha_{1}-\epsilon, \beta_{1}\right)$ and $\left(\alpha_{2}, \beta_{2}+\epsilon\right)$ are two pairs of strict lower and upper solutions of (7). Similarly to (6), let $\widetilde{T}^{*}$ be the operator corresponding to problem (7). For sufficiently large $R>0$, define

$$
\begin{aligned}
& \Omega_{\alpha_{1} \beta_{1}}=\left\{u \in E_{0}, \alpha_{1}-\epsilon \prec u \prec \beta_{1},\|u\|<R\right\}, \\
& \Omega_{\alpha_{1} \beta_{2}}=\left\{u \in E_{0}, \alpha_{1}-\epsilon \prec u \prec \beta_{2}+\epsilon,\|u\|<R\right\},
\end{aligned}
$$

and

$$
\Omega_{\alpha_{2} \beta_{2}}=\left\{u \in E_{0}, \alpha_{2} \prec u \prec \beta_{2}+\epsilon,\|u\|<R\right\} .
$$

Then $\operatorname{deg}\left(\widetilde{T}^{*}, \Omega_{\alpha_{1} \beta_{1}}, 0\right)=1, \operatorname{deg}\left(\widetilde{T}^{*}, \Omega_{\alpha_{1} \beta_{2}}, 0\right)=1$ and $\operatorname{deg}\left(\widetilde{T}^{*}, \Omega_{\alpha_{2} \beta_{2}}, 0\right)=1$. Thus by the additivity property of Brouwer degree, we have $\operatorname{deg}\left(\widetilde{T}^{*}, \Omega_{\alpha_{1} \beta_{2}} \backslash\left(\bar{\Omega}_{\alpha_{1} \beta_{1}} \cup \bar{\Omega}_{\alpha_{2} \beta_{2}}, 0\right)\right)=-1$. Therefore, problem (7) has three solutions $u_{1}, u_{2}$ and $u_{3}$ with $u_{1} \in \Omega_{\alpha_{1} \beta_{1}}, u_{2} \in \Omega_{\alpha_{2} \beta_{2}}$ and $u_{3} \in \Omega_{\alpha_{1} \beta_{2}} \backslash \bar{\Omega}_{\alpha_{1} \beta_{1}} \cup \bar{\Omega}_{\alpha_{2} \beta_{2}}$. By the facts that all solutions of (7) satisfy $\left[\alpha_{1}, \beta_{2}\right]$ and are solution of (1), the proof is complete. 


\section{Three positive solutions of eigenvalue problems}

Lemma 3.1 Let (A1) hold and $u$ satisfy the following difference inequality:

$$
-\Delta(\phi(\Delta u(k-1))) \geq 0, \quad k \in[1, T]_{\mathrm{Z}}
$$

with $u(0) \geq 0, u(T+1) \geq 0$. Then $u(k) \geq 0$ for all $k \in[1, T]_{\mathrm{Z}}$, and $\Delta u(k-1) \geq 0$ for $k \in$ $\left[1, k^{*}\right]_{\mathbf{Z}}, \Delta u(k) \leq 0$ for $k \in\left[k^{*}, T\right]_{\mathbf{Z}}$, where $k^{*} \in[0,1+T]_{\mathbf{Z}}$ satisfies $u\left(k^{*}\right)=\max _{k \in[0,1+T]_{\mathbf{Z}}} u(k)$.

Proof Since $\Delta[\phi(\Delta u(k-1))]=\phi(\Delta u(k))-\phi(\Delta u(k-1)) \leq 0, k \in[1, T]_{\mathrm{Z}}$, we have by the monotonicity of $\phi$ that $\Delta u(k) \leq \Delta u(k-1), k \in[1, T]_{\mathrm{Z}}$. If $k^{*}=0$ or $T+1$, the result is clear. Now, we assume that $k^{*} \in[1, T]_{\mathrm{z}}$. Since

$$
\begin{aligned}
& \Delta u\left(k^{*}-1\right)=u\left(k^{*}\right)-u\left(k^{*}-1\right) \geq 0, \\
& \Delta u\left(k^{*}\right)=u\left(k^{*}+1\right)-u\left(k^{*}\right) \leq 0,
\end{aligned}
$$

we have by the monotonicity of $\Delta u(\cdot)$ that $\Delta u(k-1) \geq 0$ for $k \in\left[1, k^{*}\right]_{\mathrm{Z}}, \Delta u(k) \leq 0$ for $k \in$ $\left[k^{*}, T\right]_{\mathrm{Z}}$, which implies that $u(k) \geq 0$ holds for all $k \in[1, T]_{\mathrm{Z}}$ by the boundary conditions $u(0) \geq 0, u(T+1) \geq 0$.

Remark If inequality (8) is strict, then $u(k)>0$ for $k \in[1, T]_{\mathrm{Z}}$, and there exists $k^{*} \in[0,1+$ $T]_{\mathrm{Z}}$ such that $u\left(k^{*}\right)=\|u\|$, and $\Delta u(k-1)>0$ for $k \in\left[1, k^{*}-1\right]_{\mathrm{Z}}, \Delta u\left(k^{*}-1\right) \geq 0$, and $\Delta u(k)<$ 0 for $k \in\left[k^{*}, T\right]_{\mathrm{Z}}$.

Consider the following problem:

$$
\left\{\begin{array}{l}
\Delta(\phi(\Delta u(k-1)))+h(k)=0, \quad k \in[1, T]_{\mathrm{Z}} \\
u(0)=u(T+1)=0
\end{array}\right.
$$

where $h:[1, T]_{\mathrm{Z}} \rightarrow(0, \infty)$.

In the following arguments, we assume that

(B1) $\phi: \mathbf{R} \rightarrow \mathbf{R}$ is an odd and strictly increasing homeomorphism.

Lemma 3.2 Let (B1) hold and $u$ solve (9). If h is symmetric on $[1, T]_{\mathrm{Z}}$, i.e., $h(k)=h(T+1-k)$, $k \in[1, T]_{\mathrm{Z}}$, then $u(k)$ is symmetric on $[1, T]_{\mathrm{Z}}$. Moreover,

(i) if $T+1(T \geq 2)$ is odd, then $\|u\|=u\left(\frac{T}{2}\right)=u\left(\frac{T}{2}+1\right)$, and the solution $u$ of (9) can be expressed as

$$
u(k)= \begin{cases}\sum_{s=1}^{k} \phi^{-1}\left(\sum_{l=s}^{\frac{T}{2}} h(l)\right), & k \leq \frac{T}{2} \\ \sum_{s=k}^{T} \phi^{-1}\left(\sum_{l=\frac{T}{2}+1}^{s} h(l)\right), & k \geq \frac{T}{2}+1\end{cases}
$$

(ii) if $T+1(T \geq 3)$ is even, then $\|u\|=u\left(\frac{T+1}{2}\right)$, and the solution $u$ of $(9)$ can be expressed as

$$
u(k)= \begin{cases}\sum_{s=1}^{k} \phi^{-1}\left(\sum_{l=s}^{\frac{T+1}{2}-1} h(l)+\frac{1}{2} h\left(\frac{T+1}{2}\right)\right), & k \leq \frac{T+1}{2}, \\ \sum_{s=k}^{T} \phi^{-1}\left(\sum_{l=\frac{T+1}{2}+1}^{s} h(l)+\frac{1}{2} h\left(\frac{T+1}{2}\right)\right), & k \geq \frac{T+1}{2} .\end{cases}
$$


Proof It is easy to see that

$$
u(k)=\sum_{s=1}^{k} \phi^{-1}\left(-\phi(u(T))+\sum_{l=s}^{T} h(l)\right), \quad k \in[0, T+1]_{\mathrm{Z}}
$$

with

$$
\sum_{k=1}^{T+1} \phi^{-1}\left(-\phi(u(T))+\sum_{l=k}^{T} h(l)\right)=0
$$

Equivalently,

$$
u(k)=\sum_{s=k}^{T} \phi^{-1}\left(-\phi(u(1))+\sum_{l=1}^{s} h(l)\right), \quad k \in[0, T+1]_{\mathrm{Z}},
$$

with

$$
\sum_{k=0}^{T} \phi^{-1}\left(-\phi(u(1))+\sum_{l=1}^{k} h(l)\right)=0 \text {. }
$$

By (10) or (12), one has

$$
\phi(u(1))+\phi(u(T))=\sum_{l=1}^{T} h(l)
$$

The symmetry of $h$ first implies that $u(1)=u(T)$. In fact, by (11),

$$
\begin{aligned}
0 & =\sum_{k=1}^{T+1} \phi^{-1}\left(-\phi(u(T))+\sum_{l=k}^{T} h(l)\right) \\
& =\sum_{k=1}^{T+1} \phi^{-1}\left(-\phi(u(T))+\sum_{l=1}^{T+1-k} h(l)\right) \\
& =\sum_{k=0}^{T} \phi^{-1}\left(-\phi(u(T))+\sum_{l=1}^{k} h(l)\right) .
\end{aligned}
$$

Since $\phi^{-1}$ is a homeomorphism from $\mathbf{R}$ onto itself, the solution $C$ of the equation $\sum_{k=1}^{T+1} \phi^{-1}\left(C+\sum_{l=k}^{T} h(l)\right)=0$ is unique. Comparing the equation above with (13), we have $\phi(u(1))=\phi(u(T))$. Thus for $k \in[1, T]_{\mathbf{Z}}$,

$$
\begin{aligned}
u(k) & =\sum_{s=k}^{T} \phi^{-1}\left(-\phi(u(1))+\sum_{l=1}^{s} h(l)\right)=\sum_{s=k}^{T} \phi^{-1}\left(-\phi(u(T))+\sum_{l=T+1-s}^{T} h(l)\right) \\
& =\sum_{m=1}^{T+1-k} \phi^{-1}\left(-\phi(u(T))+\sum_{l=m}^{T} h(l)\right)=u(T+1-k),
\end{aligned}
$$

the solution $u$ of $(9)$ is symmetric on $[1, T]_{\mathrm{z}}$. 
(i) Assume that $T+1(T \geq 2)$ is odd. Since $u(1)=u(T)$, by the symmetry of $h$ and (14), we have

$$
\phi(u(1))=\phi(u(T))=\sum_{l=1}^{\frac{T}{2}} h(l)=\sum_{l=\frac{T}{2}+1}^{T} h(l)
$$

Then for $k \leq \frac{T}{2}$,

$$
\begin{aligned}
u(k) & =\sum_{s=1}^{k} \phi^{-1}\left(-\phi(u(T))+\sum_{l=s}^{T} h(l)\right)=\sum_{s=1}^{k} \phi^{-1}\left(-\sum_{l=\frac{T}{2}+1}^{T} h(l)+\sum_{l=s}^{T} h(l)\right) \\
& =\sum_{s=1}^{k} \phi^{-1}\left(\sum_{l=s}^{\frac{T}{2}} h(l)\right)
\end{aligned}
$$

and for $k \geq \frac{T}{2}+1$,

$$
\begin{aligned}
u(k) & =\sum_{s=k}^{T} \phi^{-1}\left(-\phi(u(1))+\sum_{l=1}^{s} h(l)\right)=\sum_{s=k}^{T} \phi^{-1}\left(-\sum_{l=1}^{\frac{T}{2}} h(l)+\sum_{l=1}^{s} h(l)\right) \\
& =\sum_{s=k}^{T} \phi^{-1}\left(\sum_{l=\frac{T}{2}+1}^{s} h(l)\right) .
\end{aligned}
$$

Clearly, $\|u\|=u\left(\frac{T}{2}\right)=u\left(\frac{T}{2}+1\right)$.

(ii) If $T+1(T \geq 3)$ is even, then (14) and the symmetry of $h$ imply that

$$
\phi(u(1))=\phi(u(T))=\sum_{l=1}^{\frac{T+1}{2}-1} h(l)+\frac{1}{2} h\left(\frac{T+1}{2}\right)=\sum_{l=\frac{T+1}{2}+1}^{T} h(l)+\frac{1}{2} h\left(\frac{T+1}{2}\right) .
$$

Thus for $k \leq \frac{T+1}{2}$,

$$
\begin{aligned}
u(k) & =\sum_{s=1}^{k} \phi^{-1}\left(-\phi(u(T))+\sum_{l=s}^{T} h(l)\right) \\
& =\sum_{s=1}^{k} \phi^{-1}\left(-\sum_{l=\frac{T+1}{2}+1}^{T} h(l)-\frac{1}{2} h\left(\frac{T+1}{2}\right)+\sum_{l=s}^{T} h(l)\right) \\
& =\sum_{s=1}^{k} \phi^{-1}\left(\sum_{l=s}^{\frac{T+1}{2}} h(l)-\frac{1}{2} h\left(\frac{T+1}{2}\right)\right) \\
& =\sum_{s=1}^{k} \phi^{-1}\left(\sum_{l=s}^{\frac{T+1}{2}-1} h(l)+\frac{1}{2} h\left(\frac{T+1}{2}\right)\right)
\end{aligned}
$$


and for $k \geq \frac{T+1}{2}$,

$$
\begin{aligned}
u(k) & =\sum_{s=k}^{T} \phi^{-1}\left(-\phi(u(1))+\sum_{l=1}^{s} h(l)\right) \\
& =\sum_{s=k}^{T} \phi^{-1}\left(-\sum_{l=1}^{\frac{T+1}{2}-1} h(l)-\frac{1}{2} h\left(\frac{T+1}{2}\right)+\sum_{l=1}^{s} h(l)\right) \\
& =\sum_{s=k}^{T} \phi^{-1}\left(\sum_{l=\frac{T+1}{2}}^{s} h(l)-\frac{1}{2} h\left(\frac{T+1}{2}\right)\right) \\
& =\sum_{s=k}^{T} \phi^{-1}\left(\sum_{l=\frac{T+1}{2}+1}^{s} h(l)+\frac{1}{2} h\left(\frac{T+1}{2}\right)\right) .
\end{aligned}
$$

Clearly, $\|u\|=u\left(\frac{T+1}{2}\right)$. The proof is complete.

Now, we state the existence result of at least three positive solutions of (2). Throughout the following arguments, we suppose that $T \geq 4$. Let $v$ be the unique positive solution of the following boundary value problem:

$$
\left\{\begin{array}{l}
\Delta(\phi(\Delta u(k-1)))+p(k)=0, \quad k \in[1, T]_{\mathrm{Z}} \\
u(0)=u(T+1)=0
\end{array}\right.
$$

and $p_{0}=\min _{k \in[1, T] z} p(k)$.

We make the following assumptions.

(B2) There exists an increasing homeomorphism $\psi:(0, \infty) \rightarrow(0, \infty)$ such that for all $\mu>1$,

$$
\frac{\phi(\mu x)-\phi(\mu y)}{\phi(x)-\phi(y)} \geq \psi(\mu), \quad \forall x, y \in \mathbf{R}: x<y
$$

(B3) $p:[1, T]_{\mathbf{Z}} \rightarrow(0, \infty)$;

(B4) $g \in C([0, \infty),(0, \infty))$ and $\lim _{u \rightarrow \infty} \frac{g(u)}{\psi(u(u\|\|)}=0$;

(B5) There exist $a, b$ and $M$ satisfying $\|v\|<a<b<M$ such that $g$ is nondecreasing on $[b, M)$ and

$$
\max \left\{\frac{2 \phi(b) g^{*}(a)}{p_{0} g(b) \psi\left(\frac{a}{\|v\|}\right)}, \frac{T \phi(b)}{\phi\left(\frac{2 M}{T+1}\right)}\right\}<1 .
$$

Here $g^{*}(u)=\max _{0 \leq s \leq u} g(s)$.

We denote that condition (B4) implies that $\lim _{u \rightarrow \infty} \frac{g^{*}(u)}{\psi\left(\frac{u}{\|v\|}\right)}=0$ (see [12], Lemma 2.8). Clearly, $g^{*}$ is nondecreasing on $[0, \infty)$.

Assumption (B2) is satisfied by two important cases $\phi(x)=x$ and $\phi(x)=|x|^{p-2} x(p>1)$. We also provide the following two functions as examples:

$$
\phi(x)=c_{1} x^{3}+c_{2} x, \quad \phi(x)=c_{3} x^{\frac{1}{3}}+c_{4} x^{\frac{1}{5}},
$$

where $c_{i}>0(i=1,2,3,4)$. 
Theorem 3.1 Let (B1)-(B5) hold. Then for $\lambda \in\left(\lambda_{1}, \lambda_{2}\right)$, problem (2) has at least three positive solutions. Here

$$
\lambda_{1}=\frac{2 \phi(b)}{p_{0} g(b)}, \quad \lambda_{2}=\min \left\{\frac{\psi\left(\frac{a}{\|v\|}\right)}{g^{*}(a)}, \frac{2 \phi\left(\frac{2 M}{T+1}\right)}{T p_{0} g(b)}\right\}
$$

Proof Let $\lambda$ be fixed with $\lambda \in\left(\lambda_{1}, \lambda_{2}\right)$. Clearly, $\alpha_{1} \equiv 0$ is a strict lower solution of (2). Let $\beta_{1}=\frac{a}{\|v\|} v$. Note that $a>\|v\|$ and $\Delta v(k)<\Delta v(k-1), k \in[1, T]_{\mathrm{Z}}$. Then by (B2) and the monotonicity of $g^{*}$, for $k \in[1, T]_{\mathrm{Z}}$, we have

$$
\begin{aligned}
\Delta\left(\phi\left(\Delta \beta_{1}(k-1)\right)\right) & =\phi\left(\Delta \beta_{1}(k)\right)-\phi\left(\Delta \beta_{1}(k-1)\right) \\
& =\phi\left(\frac{a}{\|v\|} \Delta v(k)\right)-\phi\left(\frac{a}{\|v\|} \Delta v(k-1)\right) \\
& \leq \psi\left(\frac{a}{\|v\|}\right)(\phi(\Delta v(k))-\phi(\Delta v(k-1))) \\
& =-\psi\left(\frac{a}{\|v\|}\right) p(k) \\
& <-\lambda g^{*}(a) p(k) \\
& \leq-\lambda g^{*}\left(\beta_{1}(k)\right) p(k) \\
& \leq-\lambda g\left(\beta_{1}(k)\right) p(k) .
\end{aligned}
$$

Thus $\beta_{1}$ is a strict upper solution of (2). Now, let $\alpha_{2}$ solve the following problem:

$$
\left\{\begin{array}{l}
\Delta(\phi(\Delta u(k-1)))+\lambda^{*} p_{0} g(b)=0, \quad k \in[1, T]_{\mathrm{Z}} \\
u(0)=u(T+1)=0
\end{array}\right.
$$

where $\lambda^{*} \in\left(\lambda_{1}, \lambda\right)$. By the expression (12), we have

$$
\begin{aligned}
\alpha_{2}(1) & =\sum_{s=1}^{T} \phi^{-1}\left(-\phi\left(\alpha_{2}(1)\right)+s \lambda^{*} p_{0} g(b)\right) \\
& >\phi^{-1}\left(-\phi\left(\alpha_{2}(1)\right)+\lambda^{*} p_{0} g(b)\right),
\end{aligned}
$$

which implies that $\phi\left(\alpha_{2}(1)\right)>\frac{1}{2} \lambda^{*} p_{0} g(b)>\phi(b)$. Thus Lemma 3.2 implies that $\alpha_{2}(T)=$ $\alpha_{2}(1)>b$. Consequently, by Lemma 3.1, $\alpha_{2}(k)>b$ for all $k \in[1, T]_{\mathrm{Z}}$. Again by Lemma 3.2, one can see that if $T+1$ is odd, then

$$
\left\|\alpha_{2}\right\|=\sum_{s=1}^{\frac{T}{2}} \phi^{-1}\left(\sum_{l=s}^{\frac{T}{2}} \lambda^{*} p_{0} g(b)\right)<\frac{T+1}{2} \phi^{-1}\left(\frac{T}{2} \lambda^{*} p_{0} g(b)\right)<M,
$$

and that if $T+1$ is even, then

$$
\left\|\alpha_{2}\right\|=\sum_{s=1}^{\frac{T+1}{2}} \phi^{-1}\left(\sum_{l=s}^{\frac{T+1}{2}-1} \lambda^{*} p_{0} g(b)+\frac{1}{2} \lambda^{*} p_{0} g(b)\right)<\frac{T+1}{2} \phi^{-1}\left(\frac{T}{2} \lambda^{*} p_{0} g(b)\right)<M .
$$


Thus $b<\alpha_{2}(k)<M$ for $k \in[1, T]_{\mathbf{z}}$. Therefore,

$$
\begin{aligned}
0 & =\Delta\left(\phi\left(\Delta \alpha_{2}(k-1)\right)\right)+\lambda^{*} p_{0} g(b) \\
& \leq \Delta\left(\phi\left(\Delta \alpha_{2}(k-1)\right)\right)+\lambda^{*} p_{0} g\left(\alpha_{2}(k)\right) \\
& <\Delta\left(\phi\left(\Delta \alpha_{2}(k-1)\right)\right)+\lambda p(k) g\left(\alpha_{2}(k)\right), \quad k \in[1, T]_{\mathbf{Z}}
\end{aligned}
$$

which implies that $\alpha_{2}$ is a strict lower solution of (2). It is easy to see that

$$
\alpha_{2}(k)>b>a \geq \beta_{1}(k), \quad k \in[1, T]_{\mathrm{z}}
$$

By $\lim _{u \rightarrow \infty} \frac{g^{*}(u)}{\psi\left(\frac{u}{\|v\|}\right)}=0$, one can choose a sufficiently large positive number $C_{\lambda}$, such that

$$
\frac{g^{*}\left(\lambda C_{\lambda}\right)}{\psi\left(\frac{\lambda C_{\lambda}}{\|v\|}\right)}<\frac{1}{\lambda}
$$

and

$$
\frac{\lambda C_{\lambda}}{\|v\|}>1, \quad \alpha_{2} \leq \beta_{2}
$$

where $\beta_{2}=\lambda C_{\lambda} \frac{v}{\|v\|}$. Then by (B2) and the monotonicity of $g^{*}, \beta_{2}$ is a strict upper solution of (2). In fact, for $k \in[1, T]_{\mathrm{z}}$,

$$
\begin{aligned}
\Delta\left(\phi\left(\Delta \beta_{2}(k-1)\right)\right) & =\phi\left(\Delta \beta_{2}(k)\right)-\phi\left(\Delta \beta_{2}(k-1)\right) \\
& =\phi\left(\frac{\lambda C_{\lambda}}{\|v\|} \Delta v(k)\right)-\phi\left(\frac{\lambda C_{\lambda}}{\|v\|} \Delta v(k-1)\right) \\
& \leq \psi\left(\frac{\lambda C_{\lambda}}{\|v\|}\right)(\phi(\Delta v(k))-\phi(\Delta v(k-1))) \\
& =-\psi\left(\frac{\lambda C_{\lambda}}{\|v\|}\right) p(k) \\
& <-\lambda g^{*}\left(\lambda C_{\lambda}\right) p(k) \\
& \leq-\lambda g^{*}\left(\beta_{2}(k)\right) p(k) \\
& \leq-\lambda g\left(\beta_{2}(k)\right) p(k) .
\end{aligned}
$$

Thus by Theorem 2.1, problem (2) has three positive solutions for $\lambda \in\left(\lambda_{1}, \lambda_{2}\right)$.

Remark If $g$ is nondecreasing on $[0, \infty)$, then we take $M=\infty$ and $\lambda_{2}=\frac{\psi\left(\frac{a}{\|v\|}\right)}{g(a)}$.

\section{An example}

Taking $\phi(u)=u^{\frac{1}{3}}+u^{\frac{1}{5}}, g(u)=(u+1)^{\frac{1}{2}}, p(k) \equiv 1, T=4$, consider

$$
\left\{\begin{array}{l}
\Delta\left((\Delta u(k-1))^{\frac{1}{3}}+(\Delta u(k-1))^{\frac{1}{5}}\right)+\lambda(u(k)+1)^{\frac{1}{2}}=0, \quad k \in[1, T]_{\mathrm{Z}} \\
u(0)=u(5)=0
\end{array}\right.
$$


Let $\psi(u)=u$. It is easy to see that (B1)-(B4) hold. Choose $a=14, b=15$, then (B5) is satisfied. In fact, after some simple calculations, we get that $\|v\| \approx 1.089$ and that

$$
\begin{aligned}
& \lambda_{1}=\frac{2 \phi(b)}{p_{0} g(b)}=\frac{1}{2}\left(15^{\frac{1}{3}}+15^{\frac{1}{5}}\right) \approx 2.091, \\
& \lambda_{2}=\frac{\psi\left(\frac{a}{\|v\|}\right)}{g(a)}=\frac{14}{\sqrt{15}\|v\|} \approx 3.319 .
\end{aligned}
$$

Thus by Theorem 3.1, problem (15) has at least three positive solutions for $\lambda: 2.091<\lambda<$ 3.319 .

\section{Competing interests}

The authors declare that they have no competing interests.

\section{Authors' contributions}

All authors jointly worked on the results, and they read and approved the final manuscript.

\section{Acknowledgements}

The authors are very grateful to the referees for their helpful comments. This research is supported partially by the Research Funds for the Doctoral Program of Higher Education of China (No. 20104410120001, 201144101 10002), PCSIRT of China (No. IRT1226) and the Natural Science Fund of China (No. 11171078).

\section{Received: 5 June 2013 Accepted: 22 August 2013 Published: 8 September 2013}

\section{References}

1. Kim, C: The three-solutions theorem for $p$-Laplacian boundary value problems. Nonlinear Anal. 75, 924-931 (2012)

2. Kim, C, Shi, J: Global continuum and multiple positive solutions to a $p$-Laplacian boundary value problem. Electron. J. Differ. Equ. 106, 1-12 (2012)

3. Agarwal, RP, O'Regan, D: Boundary value problems for discrete equations. Appl. Math. Lett. 10(4), 83-89 (1997)

4. Henderson, J, Thompson, HB: Existence of multiple solutions for second-order discrete boundary value problems. Comput. Math. Appl. 43, 1239-1248 (2002)

5. Rachunkova, I, Rachunek, L: Solvability of discrete Dirichlet problem via lower and upper functions method. J. Differ Equ. Appl. 13(5), 423-429 (2007)

6. Bai, D, Xu, Y: Nontrivial solutions of boundary value problems of second order difference equations. J. Math. Anal. Appl. 326(1), 297-302 (2007)

7. Bereanu, C, Mawhin, J, Neuve, L: Existence and multiplicity results for nonlinear second order difference equations with Dirichlet value conditions. Math. Bohem. 2, 145-160 (2006)

8. Agarwal, RP, Perera, K, O'Regan, D: Multiple positive solutions of singular discrete $p$-Laplacian problems via variational methods. Adv. Differ. Equ. 2, 93-99 (2005)

9. Cabada, A, lannizzotto, A, Tersianc, S: Multiple solutions for discrete boundary value problems. J. Math. Anal. Appl. 356, 418-428 (2009)

10. Jiang, D, Pang, P, Agrwal, RP: Upper and lower solutions method and a superlinear singular discrete boundary value problem. Dyn. Syst. Appl. 16, 743-754 (2007)

11. Dang, H, Oppenheimer, S: Existence and uniqueness results for some nonlinear boundary value problems. J. Math. Anal. Appl. 198, 35-48 (1996)

12. Wang, H: On the number of positive solutions of nonlinear systems. J. Math. Anal. Appl. 281, 287-306 (2003)

13. Lee, E, Lee, Y: A multiplicity result for generalized Laplacian system with multiparameters. Nonlinear Anal. 71, e366-e376 (2009)

14. Cabada, A, Habets, P, Pouso, R: Optimal existence conditions for $\phi$-Laplacian equations with upper and lower solutions in the reversed order. J. Differ. Equ. 166, 385-401 (2000)

15. Cabada, A, Pouso, RL: Existence results for the problem $\left(\phi\left(u^{\prime}\right)\right)^{\prime}=f\left(t, u, u^{\prime}\right)$ with nonlinear boundary conditions. Nonlinear Anal. 35, 221-231 (1999)

16. Arrázola, E, Ubilla, P: Positive solutions for the 1-dimensional generalized $p$-Laplacian involving a real parameter. Proyecciones 17, 189-200 (1998)

17. Henderson, J, Wang, H: An eigenvalue problem for quasilinear systems. Rocky Mt. J. Math. 37, $215-228$ (2007)

18. Lian, W, Wong, F: Existence of positive solutions for higher order generalized p-Laplacian BVPs. Appl. Math. Lett. 13, 35-43 (2000)

19. Bai, D, Chen, Y: Three positive solutions for a generalized Laplacian boundary value problem with a parameter. Appl. Math. Comput. 219, 4782-4788 (2013)

20. Cabada, A: Extremal solutions for the difference $\phi$-Laplacian problem with nonlinear functional boundary conditions. Comput. Math. Appl. 42, 593-601 (2001)

21. Cabada, A, Otero-Espinar, V: Existence and comparison results for difference $\phi$-Laplacian boundary value problems with lower and upper solutions in reverse order. J. Math. Anal. Appl. 267, 501-521 (2002)

22. Bondar, K, Borkar, V, Patil, S: Existence and uniqueness results for difference $\phi$-Laplacian boundary value problems. ITB J. Sci. 43A(1), 51-58 (2011) 
doi:10.1186/1687-1847-2013-267

Cite this article as: Bai and Xu: Existence and multiplicity of difference $\phi$-Laplacian boundary value problems.

Advances in Difference Equations 2013 2013:267.

Submit your manuscript to a SpringerOpen ${ }^{\circ}$ journal and benefit from:

- Convenient online submission

- Rigorous peer review

- Immediate publication on acceptance

- Open access: articles freely available online

- High visibility within the field

- Retaining the copyright to your article

Submit your next manuscript at $\gg$ springeropen.com 